\title{
Adaptive Power and Rate Transmission in hybrid DS/FH CDMA Communications over Fading Channels
}

\author{
Ye Hoon Lee and Dong Ho Kim* \\ Department of Electronic and IT Media Engineering, Seoul National University of \\ Science and Technology, Seoul 139-743, Korea. \{y.lee, dongho.kim\}@snut.ac.kr
}

\begin{abstract}
We consider a hybrid direct-sequence frequency-hopped (DS/FH) code division multiple access (CDMA) communication system, where the transmission power and data rate are adapted relative to the channel variation. Instead of random frequency hopping, hopping pattern is adaptively adjusted to obtain the maximum channel gain among available frequency slots. Transmission power and/or data rate are also adapted such that a target transmission quality is maintained. It is shown that the proposed scheme provides a higher average data rate than pure DS/CDMA with power and rate adaptations, subject to the identical average transmission power and bandwidth constraints.
\end{abstract}

Keywords: hybrid DS/FH, adaptive systems, Rayleigh fading.

\section{Introduction}

The radio link can be characterized by time-varying multipath fading which causes the link quality to vary with time. When the transmitter is provided with the channel characteristics, the transmission schemes can be adapted to it, allowing the channel to be used more efficiently. Optimal adaptation of the transmission power was considered in [1] and together with peak and average power constraints in [2]. Adaptive variation of data rate [3], constellation size [4] [5], coding scheme [6], and any combination of these parameters [7]-[10] were studied, all for narrow-band systems.

In current code division multiple access (CDMA) cellular systems, open loop and closed loop power control techniques are employed in adjusting the transmission power of each mobile [11][12]. The power adaptation, however, requires a large amount of transmission power to compensate for deep fades. It was shown in [13] that in direct-sequence (DS) CDMA systems, rate adaptation with fixed transmission power provides a higher average data rate than the power adaptation with fixed data rate, when the average transmission powers and qualityof-service (QoS) requirements are identical. An optimal rate adaptation scheme with perfect power control and combined rate and power adaptation schemes

\footnotetext{
* Dong Ho Kim is the corresponding author (E-mail: dongho.kim@snut.ac.kr).
} 
were studied in [14] and [15], respectively, all for pure DS/CDMA communication systems.

In this paper, we consider combined power and rate adaptation schemes in hybrid direct-sequence frequency-hopped (DS/FH) CDMA communication systems. Instead of conventional random frequency hopping, the hopping pattern is adaptively adjusted to obtain the maximum channel gain among available frequency slots. Transmission power and/or data rate (i.e. spreading gain) are also adapted such that a target transmission quality is maintained. We analyze the proposed adaptation scheme, and compare the performance with that of the pure DS/CDMA communication systems with power and rate adaptations. Our results show that the proposed scheme provides a higher average data rate than the pure DS systems with power and rate adaptations for given average transmission power and bandwidth constraints.

The paper is organized as follows. In Section 2, we introduce the system model considered in this paper. Power and/or rate adaptations in hybrid DS/FH CDMA systems are analyzed in Section 3. In Section 4, we present numerical results and discuss the performance improvements provided by proposed adaptation schemes.

\section{System Model}

We consider a hybrid DS/FH communication system with adaptive frequency hopping in the reverse link of a CDMA cellular system. The user's binary data $d_{k}(t)$ is spread by random binary spreading sequence $p_{k}(t)$, and modulated with carrier frequency $f_{k}(t)$. The $f_{k}(t)$ is adaptively determined by the $\mathrm{FH}$ controller such that the maximum possible channel gain is attained. The FH controller receives channel state information (CSI) from the base station (BS) through a feedback channel. We assume the BS can estimate the CSI of each user perfectly. We look only at a single cell system. The implications of a multiple cell system can be accounted for by the out-of-cell interference coefficient [11]. We assume that there are $K$ users in the system, and each non-reference-user signal is misaligned relative to the reference signal by an amount $\tau_{k}, k=1,2, \cdots, K$, which is uniformly distributed over a bit interval. There are $q$ available frequency slots. Each frequency slot is assumed to be frequency-nonselective and the channel variation due to multipath fading is slow relative to the bit duration. We assume that the multipath fading is characterized by Rayleigh probability density function (pdf). The power gain on $m^{\text {th }}$ frequency slot for user $k$ is given by

$$
P_{G_{k, m}}(x)=\frac{1}{\Omega_{h}} e^{-x / \Omega_{h}}, m=1,2, \cdots, q .
$$

The received signal $y(t)$ at the BS can be represented by

$$
y(t)=\sum_{k=1}^{K_{h}+1} \sqrt{2 G_{k}^{(1)} S_{k}} d_{k}\left(t-\tau_{k}\right) p_{k}\left(t-\tau_{k}\right) \cdot \cos \left[2 \pi f_{k}\left(t-\tau_{k}\right)+\theta_{k}\right]+n(t),
$$


where $K_{h}$ is the number of hit users transmitting data over the same frequency slot as target user's, and $S_{k}$ is the transmission power of user $k . \tau_{k}$ and $\theta_{k}$ represent the path delay and the phase, respectively, for user $k$, and they are assumed independent and uniformly distributed, the former over a bit interval and the latter over $[0,2 \pi] . n(t)$ represents the zero-mean white Gaussian noise with two-sided power spectral density $N_{0} / 2 . G_{k}^{(1)}$ is the channel power gain for user $k$ after frequency dehopping. Since the hopping is adaptively adjusted such that the maximum channel gain is attained, $G_{k}^{(1)}=\max \left(G_{k, 1}, G_{k, 2}, \cdots, G_{k, q}\right)$. The pdf of $G_{k}^{(1)}$ can be given by Order Statistics [16]:

$$
P_{G_{k}^{(1)}}(x)=\frac{q}{\Omega_{h}} e^{-x / \Omega_{h}}\left(1-e^{-x / \Omega_{h}}\right)^{q-1} .
$$

The signal-to-interference ratio $E_{b} / N_{e}$ at the coherent correlation receiver output for user $i$ is given by

$$
E_{b} / N_{e}=\frac{G_{i}^{(1)} S_{i} T_{i}}{\sum_{k=1}^{K_{h}} 2 G_{k}^{(1)} S_{k} T_{c}^{h} / 3+N_{0}},
$$

where $T_{i}$ is the bit duration for user $i$, and $T_{c}^{h}$ is the chip duration. We assume $T_{c}^{h}$ is equal to the delay spread of the channel $T_{m}$, which means the required bandwidth of the hybrid DS/FH system is given by $q / T_{c}^{h}$.

\section{Power and Rate Adaptations in hybrid DS/FH CDMA}

It follows from (4) that in order to maintain the required QoS which depends on $E_{b} / N_{e}$, the information rate $R_{i} \triangleq 1 / T_{i}$ and the transmission power $S_{i}$ of user $i$ should be given by

$$
R_{i}=\frac{1}{\left(E_{b} / N_{e}\right)_{o}} \cdot \frac{G_{i}^{(1)} S_{i}}{\sum_{k=1}^{K_{h}} 2 G_{k}^{(1)} S_{k} T_{c}^{h} / 3+N_{o}} .
$$

The probability of $K_{h}$ being $h$, equivalently, the probability of $h-1$ hits occurring among $K-1$ users, is given by

$$
\operatorname{Pr}\left(K_{h}=h\right)=\left(\begin{array}{c}
K-1 \\
h
\end{array}\right) p_{h}^{h}\left(1-p_{h}\right)^{K-1-h} \triangleq B\left(K-1, h, p_{h}\right)
$$

where $p_{h}$ is the probability of a hit. The probability $p_{h}$ can be given by

$$
p_{h}=\operatorname{Pr}(\text { partial hit })+\operatorname{Pr}(\text { full hit })=\frac{1}{q}(2-\Lambda(q))
$$

where $\Lambda(q)$ is the probability of two consecutive data bits being transmitted over the same frequency slot. Since the hopping pattern is not random memoryless, $\Lambda(q)$ is given by

$$
\Lambda(q)=\operatorname{Pr}\left\{f_{k}(t)=f_{k}\left(t+T_{k}\right)\right\}=q \int_{0}^{\infty} \int_{0}^{\infty} F_{\alpha, \beta}^{q-1}(x, y) f_{\alpha, \beta}(x, y) d x d y
$$


where $f_{\alpha, \beta}(\cdot, \cdot)$ and $F_{\alpha, \beta}(\cdot, \cdot)$ are the joint pdf and cumulative distribution function $(\mathrm{CDF})$ of $\alpha \triangleq G_{k, m}(t)$ and $\beta \triangleq G_{k, m}\left(t+T_{k}\right)$, respectively. $f_{\alpha, \beta}(x, y)$ is given by $[17]$

$$
f_{\alpha, \beta}(x, y)=\frac{1}{(1-\rho) \Omega_{h}^{2}} I_{0}\left(\frac{2 \sqrt{\rho x y}}{(1-\rho) \Omega_{h}}\right) e^{-\frac{x+y}{(1-\rho) \Omega_{h}}}
$$

where $I_{0}(\cdot)$ is the zero-order modified Bessel function of the first kind, and $\rho$ is the correlation factor between $\alpha$ and $\beta . \rho$ can be expressed in terms of the bit duration $T_{k}$, the mobile speed $v$, and the wavelength of the carrier frequency $\lambda$ as $\rho=J_{0}^{2}\left(2 \pi f_{d} T_{k}\right)$, where $J_{0}(\cdot)$ is the zero-order Bessel function of the first kind, and $f_{d}=v / \lambda$ is the maximum Doppler frequency shift [18]. Notice that in (7) the probability $p_{h}$ approaches to $(1 / q)(2-1 / q)$ as $\rho$ approaches 0 , the case of random memoryless hopping.

\subsection{Power Adaptation}

We consider the case where the data rate of each user is fixed at $1 / T$, i.e. $R_{i}=$ $1 / T$ for all user $i$, and the transmission power $S_{i}$ is adapted. It follows from (5) that the transmission power $S_{i}$ such that maintain the target QoS is given by

$$
S_{i}=\frac{N_{0}}{G_{i}^{(1)}}\left[T /\left(E_{b} / N_{e}\right)_{o}-2 K_{h} T_{c}^{h} / 3\right]^{-1} .
$$

Then, the average transmission power $S_{T}$ at the mobile unit is

$$
S_{T}=E\left[S_{i}\right]=\frac{\Xi(q) N_{0}}{\Omega_{h}} \sum_{h=0}^{K-1} B\left(K-1, h, p_{h}\right)\left[T /\left(E_{b} / N_{e}\right)_{o}-2 h T_{c}^{h} / 3\right]^{-1}
$$

where

$$
\Xi(q) \triangleq \Omega_{h} \int_{0}^{\infty} \frac{1}{g} P_{G_{i}^{(1)}}(g) d g=q \sum_{j=0}^{q-1}(-1)^{q+j}\left(\begin{array}{c}
q-1 \\
j
\end{array}\right) \ln (q-j) .
$$

There is no closed-form solution for the (average) data rate $\bar{R}_{i}=1 / T$ in (11). We calculate it using numerical search technique. Note that the bit duration $T$ in (10) should exceed $2\left(E_{b} / N_{e}\right)_{o} K_{h} T_{c}^{h} / 3$ in order to keep the received power to be positive (i.e. to maintain the target QoS). The (average) data rate, therefore, is limited by

$$
\bar{R}_{i}=1 / T<\left[2\left(E_{b} / N_{e}\right)_{o}(K-1) T_{c}^{h} / 3\right]^{-1} .
$$

\subsection{Combined Power and Rate Adaptation}

We now consider adapting the transmission power $S_{i}$ relative to $G_{i}^{(1)}$ such that $G_{i}^{(1)} S_{i}$ is equal to $S_{R}$. This power adaptation ensures that all mobile signals are 
received with the same constant power $S_{R}$. The data rate $R_{i}$ may be adapted to maintain target transmission quality, since the number of interference users (i.e. hit users) varies. We call this power and rate adaptation. Then, the average transmission power at the mobile is

$$
S_{T}=E\left[S_{i}\right]=S_{R} E\left[1 / G_{i}^{(1)}\right]=\Xi(q) S_{R} / \Omega_{h}
$$

It follows from (5), (6), and (14) that the average data rate $\bar{R}_{i}$ is given by

$$
\bar{R}_{i}=\frac{1}{\left(E_{b} / N_{e}\right)_{o}} \sum_{h=0}^{K-1}\left[\frac{B\left(K-1, h, p_{h}\right)}{2 h T_{c}^{h} / 3+\Xi(q) N_{o} /\left(\Omega_{h} S_{T}\right)}\right] .
$$

A lower bound on the average data rate $\bar{R}_{i}$ can be obtained by using Jensen's inequality [16]:

$$
\bar{R}_{i} \geq \frac{1}{\left(E_{b} / N_{e}\right)_{o}} \cdot \frac{1}{2 p_{h}(K-1) T_{c}^{h} / 3+\Xi(q) N_{o} /\left(\Omega_{h} S_{T}\right)} .
$$

We will see later that the lower bound is very close to the exact value.

\subsection{Rate Adaptation}

Next, we consider adapting the data rate $R_{i}$, while the transmission power of each user is fixed at $S_{T}$. It follows from (5) that the average data rate $\bar{R}_{i}$ with the rate adaptation is expressed as

$$
\bar{R}_{i}=\frac{3 \Omega_{h} \Psi(q)}{2\left(E_{b} / N_{e}\right)_{o} T_{c}^{h}} \cdot E\left[E\left[\frac{1}{I} \mid K_{h}\right]\right]
$$

where

$$
\Psi(q) \triangleq \frac{1}{\Omega_{h}} \int_{0}^{\infty} g P_{G_{i}^{(1)}}(g) d g=q \sum_{k=0}^{q-1}(-1)^{k-q+1}\left(\begin{array}{c}
q-1 \\
k
\end{array}\right) \frac{1}{(q-k)^{2}}
$$

and

$$
I \triangleq \sum_{k=1}^{K_{h}} G_{k}^{(1)}+\frac{3 N_{0}}{2 S_{T} T_{c}^{h}}
$$

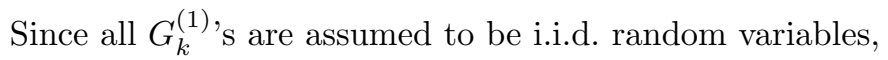

$$
E\left[\frac{1}{I} \mid K_{h}\right]=\int_{a}^{\infty} \frac{1}{x} P_{I}(x) d x=\frac{1}{2 \pi} \int_{a}^{\infty} \frac{1}{x} \int_{-\infty}^{\infty} \varphi^{K_{h}}(\omega) e^{-j \omega(x-a)} d \omega d x
$$

where

$$
a \triangleq \frac{3 N_{0}}{2 S_{T} T_{c}^{h}}
$$


Fig. 1. The average data rate versus $S_{T} / N_{0} ;\left(E_{b} / N_{e}\right)_{o}=7[\mathrm{~dB}], K=60, q=L=$ 4, $T_{m}=1[\mu \mathrm{sec}], f_{d} T_{i}=10^{-2}$.

$$
\text { figure }=\text { eta.ps, width }=8 \mathrm{~cm}
$$

Fig. 2. The average spectral efficiency versus $S_{T} / N_{0} ;\left(E_{b} / N_{e}\right)_{o}=7[\mathrm{~dB}], K=60, q=$ $L=4, T_{m}=1[\mu \mathrm{sec}], f_{d} T_{i}=10^{-2}$.

$P_{I}(x)$ is the pdf of $I$, and $\varphi(\omega)$ is the characteristic function of $G_{k}^{(1)}$, given by

$$
\varphi(\omega)=\int_{0}^{\infty} P_{G_{k}^{(1)}}(x) e^{j \omega x} d x=q \sum_{k=0}^{q-1}(-1)^{k-q+1}\left(\begin{array}{c}
q-1 \\
k
\end{array}\right) \frac{1}{\left(q-k-j \Omega_{h} \omega\right)} .
$$

Then, the average data rate is given by

$$
\bar{R}_{i}=\frac{3 \Omega_{h} \Psi(q)}{4 \pi\left(E_{b} / N_{e}\right)_{o} T_{c}^{h}} \sum_{h=0}^{K-1} B\left(K-1, h, p_{h}\right) \int_{a}^{\infty} \int_{-\infty}^{\infty} \frac{1}{x} \varphi^{h}(\omega) e^{-j \omega(x-a)} d \omega d x .
$$

\section{Numerical Results and Discussions}

The average data rates versus $S_{T} / N_{o}$ are shown in Fig. 1. We can see the lower bounds on the average data rate, (16) and (??), are virtually identical the exact values. We find that the combined hopping, rate, and power adaptations in hybrid DS/FH systems provide higher average data rates than the power or rate adaptation in pure DS systems. Notice that the average data rate with power adaptation in hybrid DS/FH system is not increased even though $S_{T} / N_{0}$ becomes higher. This is because the achievable data rate is limited by (13) in order to maintain the target QoS all the time. We find the rate adaptation in hybrid DS/FH system provides superior performance to any other adaptation schemes. This results from the facts that the rate adaptation uses a constant power and compensates for deep fades by reducing the rate rather than using a large power, and the average received power in the hybrid DS/FH system is higher than that in the pure DS system. However, the rate adaptations provide variable data rate, which is more profitable to non delay-limited services such as file transfer and email services.

If we normalize the average data rate $\bar{R}_{i}$ by the total bandwidth $1 / T_{c}^{p}$ (equivalently, $q / T_{c}^{h}$ ), then we get the average spectral efficiency in bit/sec/Hz. Fig. 2 presents the average spectral efficiencies versus bandwidth expansion factor $q(=L)$. We find that there exist an optimal bandwidth which maximizes the average spectral efficiency when the power adaptation is employed in pure DS and when the power and rate adaptation is used in hybrid DS/FH systems. In the pure DS system, decrease in $L$ reduces the average transmitted data 
rate, since the combining capability of RAKE receiver and effective spreading gain decrease. In the hybrid DS/FH system, decrease in $q$ also reduces the average data rate, since the probability of deep fading increases and the average received power decreases. However, the required bandwidth for both systems is also reduced. Thus, increasing (or decreasing) the bandwidth produces two counteracting effects on the average spectral efficiency of the system. We find the average spectral efficiencies for pure DS and hybrid DS/FH with rate adaptation are monotonic functions of the bandwidth. This indicates that when the rate adaptation is employed, the effect of reducing required bandwidth on the average spectral efficiency is crucial over all the range of system bandwidth.

\section{Acknowledgments}

This work was supported by the Basic Science Research Programs through the National Research Foundation of Korea(NRF) funded by the Ministry of Education, Science and Technology (No. 2012-0003899 and No. 2012R1A1A2044903).

\section{References}

1. Hayes, J.F.: Adaptive feedback communications. IEEE Trans. Commun. 16 (Feb. 1968) 29-34

2. Lee, Y.H., Bar-Ness, Y.: Power adaptation for BPSK signaling with average and peak power constraints in Rayleigh fading channels. IEEE Trans. Commun. 51 (Nov. 2003) 1871-1876

3. Cavers, J.K.: Variable-rate transmission for Rayleigh fading channels. IEEE Trans. Commun. 20 (Feb. 1972) 15-22

4. Webb, W.T., Steele, R.: Variable rate QAM for mobile radio. IEEE Trans. Commun. 43 (July 1995) 2223-2230

5. Kamio, Y., Sampei, S., Sasaoka, H., Morinaga, N.: Performance of modulationlevel-controlled adaptive-modulation under limited transmission delay time for land mobile communications. In: Proc. IEEE VTC. (July 1995) 221-225

6. Vucetic, B.: An adaptive coding scheme for time-varying channels. IEEE Trans. Commun. 39 (May 1991) 653-663

7. Alamouti, S.M., Kallel, S.: Adaptive trellis-coded multiple-phase-shift keying for Rayleigh fading channels. IEEE Trans. Commun. 42 (June 1994) 2305-2314

8. Ue, T., Sampei, S., Morinaga, N.: Symbol rate and modulation level controlled adaptive modulation/TDMA/TDD for personal communication systems. In: Proc. IEEE VTC. (July 1995) 306-310

9. Goldsmith, A.J., Chua, S.G.: Variable-rate variable-power MQAM for fading channels. IEEE Trans. Commun. 45 (Oct. 1997) 1218-1230

10. Chung, S.T., Goldsmith, A.J.: Degrees of freedom in adaptive modulation: a unified view. IEEE Trans. Commun. 49 (Sep. 2001) 1561-1571

11. Gilhousen, K.S., et al.: On the capacity of a cellular CDMA system. IEEE Trans. Veh. Technol. 40 (May 1991) 303-312

12. Zander, J.: Performance of optimum transmitter power control in cellular radio systems. IEEE Trans. Veh. Technol. 41 (Feb. 1992) 57-62

13. Kim, S.W.: Adaptive rate and power DS/CDMA communications in fading channels. IEEE Commun. Lett. (Apr. 1999) 85-87 
14. Oh, S.J., Wasserman, K.M.: Dynamic spreading gain control in multiservice CDMA networks. IEEE J. Select. Areas Commun. 17 (May 1999) 918-927

15. Kim, S.W., Lee, Y.H.: Combined rate and power adaptation in DS/CDMA communications over Nakagami fading channels. IEEE Trans. Commun. (Jan. 2000) $162-168$

16. Feller, W.: An Introduction to Probability Theory and Its Applications. 2nd edn. Vol. II. John Wiley and Sons (1971)

17. Schwartz, M., Bennett, W.R., Stein, S.: Communication Systems and Techniques. New-York: McGraw-Hill (1966)

18. Jakes, Jr., W.C.: Microwave Mobile Communications. John Wiley and Sons (1974) 